Check for updates

Cite this: RSC Adv., 2017, 7, 32861

Received 5th April 2017

Accepted 19th June 2017

DOI: $10.1039 / \mathrm{c} 7 \mathrm{ra} 03882 \mathrm{~h}$

rsc.li/rsc-advances

\section{Preparation of transparent monolithic methylsilsesquioxane (MSQ) aerogels via ambient pressure drying}

\author{
Tao Zhang, ${ }^{a}$ Hua Yuan, ${ }^{a}$ Shuangqing Wang, ${ }^{* a}$ Xudong Guo, ${ }^{a}$ Rui Hu, (D) ${ }^{a}$ Yi Lib \\ and Guogiang Yang (iD *a
}

Monolithic methylsilsesquioxane (MSQ) aerogels have been prepared from methyltrimethoxysilane (MTMS) via an ambient pressure drying (APD) method. Tetraethylammonium hydroxide (TEAH) was used to suppress macroscopic phase separation and accelerate the condensation reaction. The obtained hydrophobic MSQ aerogels are highly transparent and withstand exposure to aggressive media for extended periods of time. This novel anti-corrosion property can be further improved by chemical modification with POTS and PDTS.
Aerogels are generally defined as dried gels with a very high relative pore volume. ${ }^{\mathbf{1}}$ These porous solids are mostly composed of a nano network derived from the precursor solution and open mesopores occupied by gas. As a result of this unique nanostructure, these low-density materials have many fascinating properties, ${ }^{1-4}$ such as high porosity (80-99.8\%), large specific surface area (100-1000 $\mathrm{m}^{2} \mathrm{~g}^{-1}$ ), the lowest acoustic impedances of all solid materials $\left(Z=10^{4}-10^{5} \mathrm{~kg} \mathrm{~m}^{-2} \mathrm{~s}^{-1}\right)$, low thermal conductivity (0.017-0.021 $\mathrm{W} \mathrm{m} \mathrm{m}^{-1} \mathrm{~K}^{-1}, 300 \mathrm{~K}$ in air), low refractive index (1.007-1.24) and low dielectric constant (lower than 2.0). Owing to these unusual properties, aerogels have been employed in tremendous applications such as catalysts, ${ }^{5}$ acoustic insulation, ${ }^{6}$ super thermal insulation in space industry, ${ }^{7}$ Cherenkov radiation detectors, ${ }^{8}$ etc. ${ }^{9}$

Among all the aerogels, silica aerogels are the most widely investigated since they were first prepared in the 1930s via a supercritical drying (SCD) method. ${ }^{10}$ SCD method is still almost the only way to completely eliminate the large capillary force caused by the receding liquid menisci. ${ }^{11}$ However, SCD method usually involves a high-pressure process which causes safety problems especially when operating with inflammable and toxic organic solvents. ${ }^{12}$

For freeze drying, no menisci will appear during the solvent removal. ${ }^{13-16}$ However, crystallization of solvent may damage the solid nano network. ${ }^{17}$ Generally, freeze drying leads to cracked pieces or even powder-like cryogels. ${ }^{18}$

\footnotetext{
${ }^{a}$ Beijing National Laboratory for Molecular Sciences, Key Laboratory of Photochemistry, Institute of Chemistry, University of Chinese Academy of Sciences, Chinese Academy of Sciences, Beijing, 100190, P. R. China. E-mail: g1704@iccas.ac. cn; gqyang@iccas.ac.cn; Fax: +8610 82617315; Tel: +861082617263

${ }^{b}$ Key Laboratory of Photochemical Conversion and Optoelectronic Materials, Technical Institute of Physics and Chemistry, Chinese Academy of Sciences, Beijing 100190, China.E-mail: yili@mail.ipc.ac.cn
}

From an industrial manufacturing point of view, ambient pressure drying (APD) is clearly more practical. The biggest obstacle for APD is that the reactive silanol groups on the gel skeleton undergo condensation reactions which make the solid network undesirably densified. ${ }^{19}$ To overcome this problem, Brinker et al. reported the first synthesis of $\mathrm{SiO}_{2}$ aerogels by APD method, which involved a series of solvent-exchange processes and a silylation of the skeleton. ${ }^{20}$ Surface chemical modification of silica aerogels using various organosilane compounds have been widely studied since then. ${ }^{\mathbf{2 1 - 2 6}}$

Preparation of modified silica gels using organosilane species (e.g. alkyl-alkoxy silanes and bridged alkoxy silanes) as precursor or co-precursor rather than post modification is a much rapid and facile way to reduce reactivity of the surface silanols. ${ }^{27-33}$ Non-polar groups bonded to the central silicon atoms can prevent permanent shrinkage during APD, but the obtained aerogels are less transparent as a result of macroscopic phase separation in most cases. ${ }^{29,30,34-37}$ Guo et al. carefully studied gelation behavior and phase separation of macroporous methylsilsesquioxane monoliths prepared by in situ two-step processing. ${ }^{38,39}$ Kanamori et al. reported an approach using the trifunctional methyl-trimethoxysilane (MTMS) as a precursor and using a variety of surfactants as a phase-separation inhibitor to prepare transparent elastic methylsilsesquioxane (MSQ) aerogels. ${ }^{\text {40-42 }}$ As this method is only applicable to water system, it is tedious to exchange the water in the gel-pores for a water-free organic solvent before dying.

Herein, we report a simple, effective way to produce transparent monolithic MSQ aerogels from MTMS via ambient pressure drying method. The preparation of MSQ aerogels was based on a two-step procedure carried in propanol using TEAH as a gelation inducer, and no extra surfactant $\mathrm{t}^{\mathbf{4 3 , 4 4}}$ like Nonion EH-208 or CTAB was needed. The obtained MSQ aerogels are 
highly transparent and withstand exposure to aggressive media for extended periods of time. This novel anti-corrosion property can be further improved by chemical modification with perfluorooctyltrichlorosilane (POTS) and perfluorodecyltrichlorosilane (PDTS).

For the production of aerogels, a two-step procedure was applied. A reaction solution of MTMS (10.0 mL), propanol (8.0 $\mathrm{mL}), \mathrm{HF}(14 \mu \mathrm{mol})$ and deionized water $(2.0 \mathrm{~mL})$ was mixed and stirred for $1.5 \mathrm{~h}$ in the first step. As propanol promoted the mixing of the reaction species effectively, the sol kept homogenous during the whole acid-catalyzed hydrolysis step.

Then a basic solution of TEAH $(0.1 \mathrm{mmol})$, deionized water $(5.0 \mathrm{~mL})$ and propanol $(5.0 \mathrm{~mL})$ was dropwise added to the reaction mixture under vigorous stirring. The $\mathrm{pH}$ of the sol rose due to the addition of TEAH, which accelerated the condensation rate and hence gelation took place rapidly. It was observed that the temperature of the reaction mixture spontaneously increased, which was the result of silanol condensation. This in turn promoted the collisions between the colloidal particles, so that the gel formed quickly. The gelation time $\left(T_{\mathrm{g}}\right)$ for the sol with TEAH was found to be about 10 minutes at room temperature.

After aging in sealed containers for 1.5 days at $50{ }^{\circ} \mathrm{C}$, the gel samples were washed with propanol at $50{ }^{\circ} \mathrm{C}$ for $8 \mathrm{~h}$ thrice and with petroleum ether at $25{ }^{\circ} \mathrm{C}$ for $8 \mathrm{~h}$ thrice. For ambient pressure drying, the washed gels were let to evaporate at room temperature overnight and then were dried at 60, 120 and $220{ }^{\circ} \mathrm{C}$ for $3 \mathrm{~h}$ respectively in an oven. The obtained modified aerogels were named as MSQ-TEAH.

TEAH is a strong base and has a large-cation group. It can act as both a catalyst to and a peptizer to disperse nanoparticles into homogeneous colloidal form. ${ }^{45}$ TEAH effectively accelerated condensation reaction and suppressed phase separation in propanol with less water so that transparent gels were formed quickly rather than precipitation.

TEAH is soluble in organic solvents, especially in alcohols and it has a low boiling point about $102{ }^{\circ} \mathrm{C}$. Thus TEAH could be easily removed during the following solvent exchange and drying operations. It is important to remove the catalyst without high temperature treatment because the methyl groups of MSQ aerogels will breaks at high temperature (e.g. above $400{ }^{\circ} \mathrm{C}$ in atmosphere) and thus make the aerogels vulnerable to humid environment. ${ }^{25}$

Other base such as ammonia (AM, $0.1 \mathrm{mmol}$ ) and triethylamine (TEA, $0.1 \mathrm{mmol}$ ) were used to catalysis the condensation reaction as well, however, without the large-cation group they

Table 1 The gelation time $\left(T_{\mathrm{g}}\right)$ of $M S Q$ sols with different catalysts

\begin{tabular}{llll}
\hline & $T_{\mathrm{g}}(\mathrm{h})$ & & \\
\cline { 2 - 3 } Base & $\mathrm{RT}$ & $50{ }^{\circ} \mathrm{C}$ & Remarks \\
\hline TEAH & $0.1-0.2$ & - & $\begin{array}{l}\text { Transparent } \\
\text { monolithic }\end{array}$ \\
AM & 3 & 1 & $\begin{array}{l}\text { Opaque pieces } \\
\text { TEA }\end{array}$ \\
& 4 & 2 & Opaque pieces
\end{tabular}

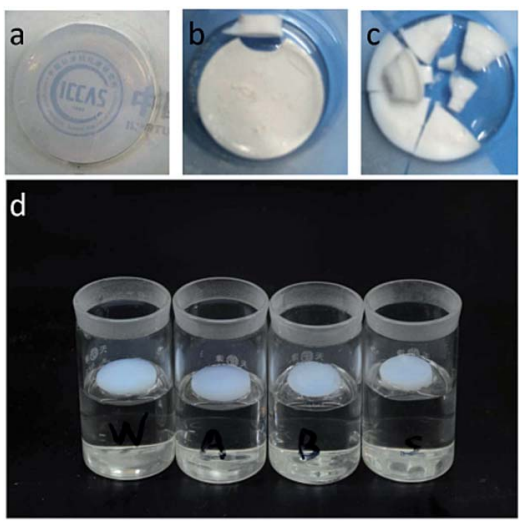

Fig. 1 Images of (a) MSQ-TEAH, (b) MSQ-AM, (c) MSQ-TEA, and (d) MSQ-TEAH aerogel samples floating on water, saltwater $(\mathrm{NaCl} 3.5 \%$ $\mathrm{w} / \mathrm{w})$, hydrochloric acid aqueous solution $(\mathrm{HCl} 1 \mathrm{M})$ and sodium hydroxide aqueous solution ( $\mathrm{NaOH} 1 \mathrm{M})$ over 3 hours.

couldn't replace the role of TEAH. Gelation time for sol with ammonia or triethylamine was much more longer. As it shown in Table 1, it took several hours to gelation for sol with ammonia and triethylamine even at higher temperature $\left(50^{\circ} \mathrm{C}\right)$. And macroscopic phase separation occurred during the sol-gel process. Consequently transparence of aerogels decreased as a result of scattering.

Moreover, differential drying shrinkage occurred when the microstructure of gels was not uniform. After APD the TEAH catalyzed MSQ aerogels (MSQ-TEAH) was monolithic and transparent while the ammonia and the triethylamine catalyzed MSQ aerogels (named as MSQ-AM and MSQ-TEA, respectively) were opaque and broke into pieces (see Fig. 1a-c).

We found that the MSQ aerogels were insensitive to various aggressive water solutions. The aerogel samples were exposed to 4 different media: water, saltwater $(\mathrm{NaCl} 3.5 \% \mathrm{w} / \mathrm{w})$, hydrochloric acid aqueous solution ( $\mathrm{HCl} 1 \mathrm{M}$ ) and sodium hydroxide aqueous solution ( $\mathrm{NaOH} 1 \mathrm{M}$ ) for 3 hours. As it shown in Fig. 1d,

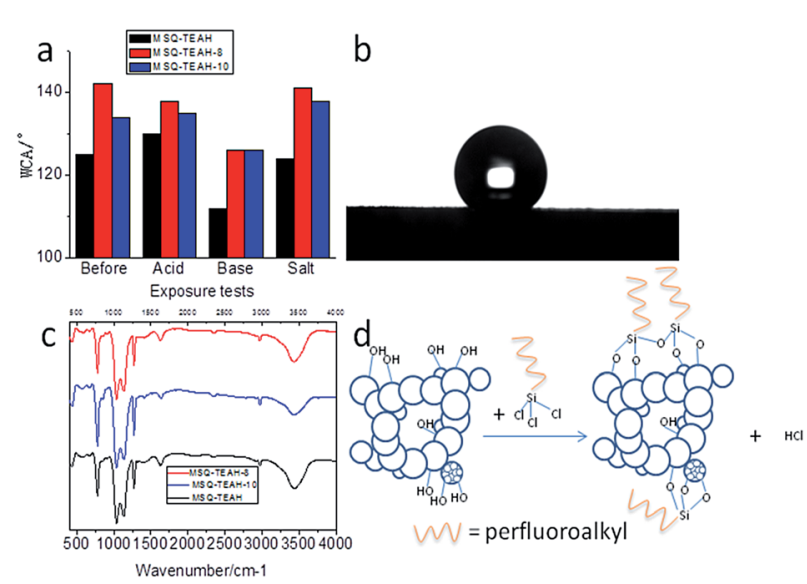

Fig. 2 (a) WCA of MSQ aerogel samples before and after exposure tests, (b) an image of a droplet on the surface of MSQ-TEAH-8 after exposed to saltwater $\left(\mathrm{WCA}=141^{\circ}\right)$, (c) FT-IR spectra of dried aerogel samples in $\mathrm{KBr}$ pellet, (d) schematic of chemical modification. 
MSQ-TEAH aerogel samples kept floating on the surface of the solutions after exposure tests. Wettability of the surface is a key feature to show anti-corrosion properties. In our study, wettability of MSQ-TEAH was evaluated by water contact angle (WCA) measurements before and after exposure tests (see Fig. 2a). Practically no change was observed during the experiment, except for aerogels exposed to the $\mathrm{NaOH}$ solution for 3 hours. However, the WCA of MSQ-TEAH after exposed to NaOH solution was above $90^{\circ}$, which indicated the hydrophobicity was maintained. ${ }^{46}$

The contact angle of the surface is closely related to the surface energy. Perfluorooctyltrichlorosilane (POTS) and perfluorodecyltrichlorosilane (PDTS) are widely used for surface modification because of their low surface energy perfluoro

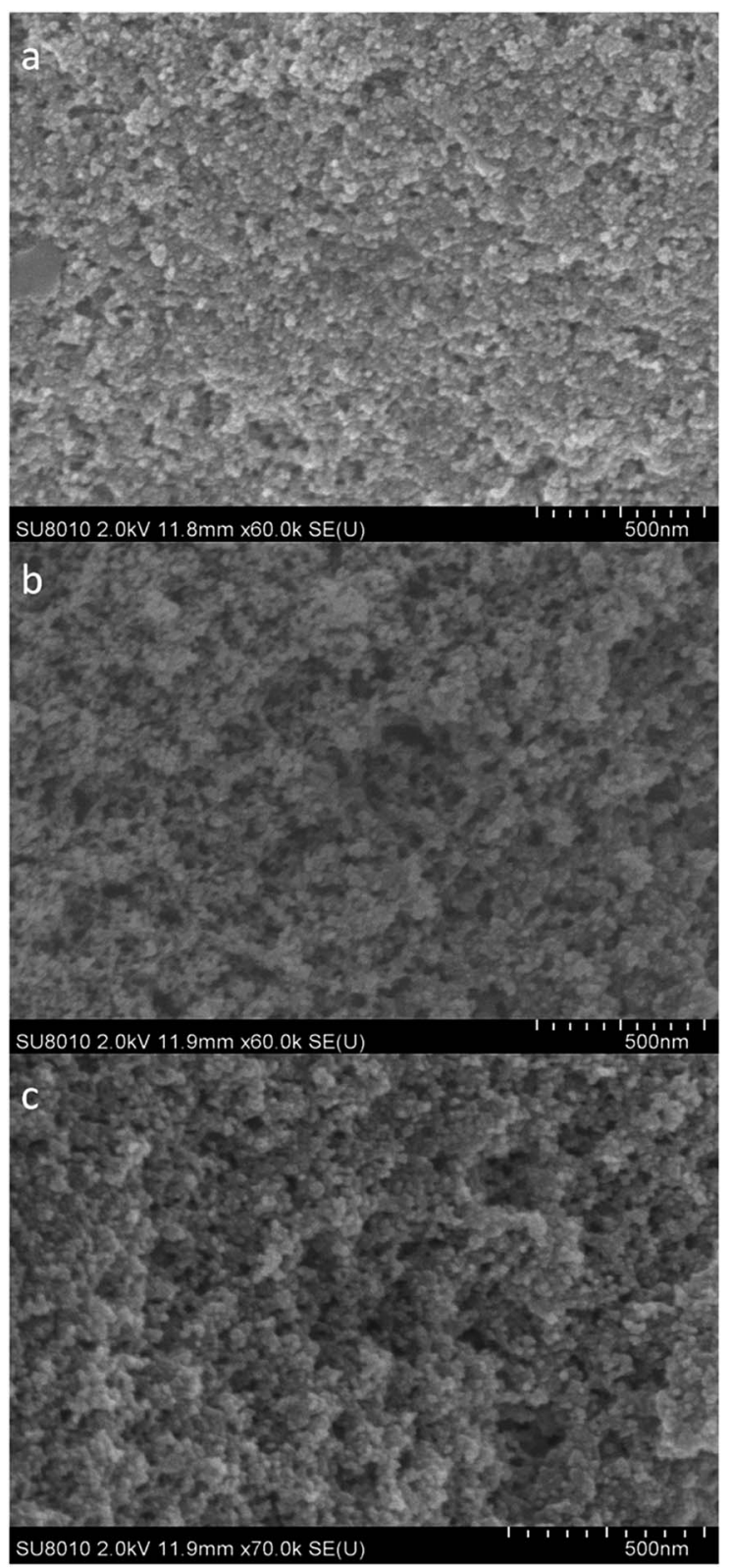

Fig. 3 SEM images of (a) MSQ-TEAH, (b) MSQ-TEAH-8, (c) MSQTEAH-10. groups grafted onto the substrate easily. ${ }^{47-51}$ Before APD drying, MSQ-TEAH gel samples were chemically modified by immersion in POTS/petroleum ether solution 1\% (v/v) and PDTS/ petroleum ether solution $1 \%(\mathrm{v} / \mathrm{v})$ for $8 \mathrm{~h}$. Schematic of chemical modification are shown in Fig. 2d. The obtained aerogels were MSQ-TEAH-8 and MSQ-TEAH-10, respectively.

The WCA before and after exposure tests were both increased (see Fig. 2a). MSQ-TEAH-8, MSQ-TEAH-8 and MSQ-TEAH-10 even showed WCAs higher than $120^{\circ}$ after exposed to $\mathrm{NaOH}$ solution for $3 \mathrm{~h}$. The WCA of MSQ-TEAH-8 after exposed to saltwater was $141^{\circ}$ (Fig. 2b), illustrating that the sample showed good stability. ${ }^{52}$ These anti-corrosion properties resulted from both low surface energy and hindrance of perfluoro and methyl groups.

The FT-IR spectra of TEAH catalyzed MSQ aerogels are shown in Fig. 2c. The broad peaks around $3400 \mathrm{~cm}^{-1}$ and peaks at $1650 \mathrm{~cm}^{-1}$ are assigned to the $\mathrm{O}-\mathrm{H}$. The peaks at $2960 \mathrm{~cm}^{-1}$ can be attributed to the absorption of $\mathrm{C}-\mathrm{H}$. The peaks at 1270 $\mathrm{cm}^{-1}$ are related to the absorption of $\mathrm{Si}-\mathrm{C}$ and $\mathrm{C}-\mathrm{H}$. The strong peaks in the $1200-1000 \mathrm{~cm}^{-1}$ region are due to the $\mathrm{Si}-\mathrm{O}-\mathrm{Si}$ absorption and the peaks at $780 \mathrm{~cm}^{-1}$ are due to the $\mathrm{O}-\mathrm{Si}-\mathrm{O}$ absorption. It's the $\mathrm{Si}-\mathrm{O}$ bonds which constitute the aerogel skeleton and the non-hydrolysable groups attaching to the backbone make the aerogels hydrophobic..$^{22,36,37,41}$

Air trapped in the nanopores also plays an important role in this anti-corrosion property, since it effectively reduced the contact area between the liquid and aerogel surface.

The morphologies of TEAH catalyzed aerogels were investigated by SEM analysis given in Fig. 3a-c. The SEM images show that all the samples have a highly porous network structure with uniform pore size.

The porosity properties of the aerogels were further revealed by the $\mathrm{N}_{2}$ adsorption and desorption isotherms. TEAH catalyzed aerogels exhibit similar type IV isotherms that are typical for mesopores (Fig. 4a). The average pore size of aerogels range between 14.1 and $18.2 \mathrm{~nm}$ (Table 2), calculated from the adsorption branch of the isotherms using the Barrett-JoynerHalenda (BJH) method. After chemical modification, a decrease of average pore size by 2-4 $\mathrm{nm}$ was observed in MSQ-TEAH-8 and MSQ-TEAH-10 due to the perfluoro alkyl groups grafted to the pore surface. Other properties like bulk density $\left(\rho_{\mathrm{b}}\right)$, skeleton density $\left(\rho_{\mathrm{s}}\right)$, porosity $(\varepsilon)$ and specific surface $\left(S_{\mathrm{BET}}\right)$ of the MSQ aerogels are also available in Table 2.
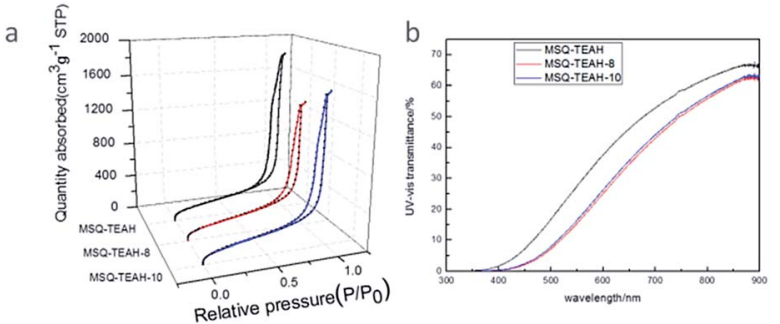

Fig. 4 (a) Adsorption and desorption isotherms of TEAH catalyzed MSQ aerogels, (b) UV-Vis spectra of TEAH catalyzed MSQ aerogels with a thickness of $5 \mathrm{~mm}$. 
Table 2 Textural properties of the MSQ aerogels

\begin{tabular}{llllll}
\hline Sample & $\rho_{\mathrm{b}}\left(\mathrm{g} \mathrm{cm}^{-3}\right)$ & $\rho_{\mathrm{s}}\left(\mathrm{g} \mathrm{cm}^{-3}\right)$ & $\varepsilon(\%)$ & $S_{\text {BET }}\left(\mathrm{m}^{2} \mathrm{~g}^{-1}\right)$ & $d(\mathrm{~nm})$ \\
\hline MSQ-TEAH & 0.159 & 1.364 & 88.3 & 559 & 18.2 \\
MSQ-TEAH-8 & 0.156 & 1.365 & 88.6 & 560 & 14.1 \\
MSQ-TEAH-10 & 0.154 & 1.346 & 88.6 & 568 & 16.0 \\
\hline
\end{tabular}

The prepared MSQ-TEAH-8 and MSQ-TEAH-10 are transparent monolithic aerogels, too. Their transparency are similar to that of MSQ-TEAH, only with a slightly reduction as shown in Fig. $4 \mathrm{~b}$.

The homogeneity of the aerogel microstructure played an important role in high light transmittance. ${ }^{53}$ The high transparency of all the TEAH catalyzed aerogels indicated that the pores and particles present in the aerogels are regular and small enough. ${ }^{54}$ In addition, the transmittance of all the TEAH catalyzed aerogels in UV region is close to zero indicating a strong scattering of short light waves.

\section{Conclusions}

In this study, a novel method for preparing transparent monolithic MSQ aerogels via ambient pressure drying is demonstrated. Tetraethylammonium hydroxide (TEAH) was used to suppress macroscopic phase separation and to accelerate the condensation reaction. The resultant MSQ-TEAH aerogels are mesoporous with an average pore size of $18.2 \mathrm{~nm}$ and a large specific surface of $559 \mathrm{~m}^{2} \mathrm{~g}^{-1}$. Besides they are highly transparent and withstand exposure to various aggressive aqueous environments for extended periods of time. This novel anti-corrosion property is attributed to the high concentration of hydrophobic groups bonded to the aerogel skeleton and air trapped in the network. After chemically modification with low surface energy silanes like PDTS and POTS, the aerogel's chemical stability has been further improved. We expect that this new TEAH-assist synthesis route of chemically stable aerogels will further greatly promote the commercial exploitation of these materials.

\section{Acknowledgements}

We are grateful for funding from the National Basic Research Program (2013CB834505 and 2013CB834703) and the National Natural Science Foundation of China (Grant No. 21627802, 91623105, 21373240 and 21233011).

\section{References}

1 N. Hüsing and U. Schubert, Angew. Chem., Int. Ed., 1998, 37, 22-45.

2 L. Hrubesh, Chem. Ind., 1990, 24, 824-827.

3 M. Aegerter, N. Leventis and M. M. Koebel, Aerogels handbook, Springer Science \& Business Media, 2011.

4 M. Chen, Z. Li, J. Li, J. Li, Q. Li and L. Zhang, Talanta, 2017, 166, 284-291.
5 G. M. Pajonk, Catal. Today, 1997, 35, 319-337.

6 L. Forest, V. Gibiat and T. Woignier, J. Non-Cryst. Solids, 1998, 225, 287-292.

7 J. E. Fesmire, Cryogenics, 2006, 46, 111-117.

8 M. Tabata, I. Adachi, H. Kawai, M. Kubo and T. Sato, Phys. Procedia, 2012, 37, 642-649.

9 A. C. Pierre and G. M. Pajonk, Chem. Rev., 2002, 102, 42434266.

10 S. S. Kistler, Nature, 1931, 127, 741.

11 A. Bisson, A. Rigacci, D. Lecomte, E. Rodier and P. Achard, Drying Technol., 2003, 21, 593-628.

12 S. Henning, in Aerogels, Springer, 1986, pp. 38-41.

13 S. Nardecchia, D. Carriazo, M. L. Ferrer, M. C. Gutierrez and F. del Monte, Chem. Soc. Rev., 2013, 42, 794-830.

14 H. W. Liang, Q. F. Guan, L. F. Chen, Z. Zhu, W. J. Zhang and S. H. Yu, Angew. Chem., Int. Ed., 2012, 51, 5101-5105.

15 X. Wang, L. L. Lu, Z. L. Yu, X. W. Xu, Y. R. Zheng and S. H. Yu, Angew. Chem., Int. Ed., 2015, 54, 2397-2401.

16 Z. Y. Wu, C. Li, H. W. Liang, J. F. Chen and S. H. Yu, Angew. Chem., Int. Ed., 2013, 52, 2925-2929.

17 G. W. Scherer, J. Non-Cryst. Solids, 1993, 155, 1-25.

18 E. Degn Egeberg and J. Engell, J. Phys., Colloq., 1989, 24, 2328.

19 L. Zhuravlev, Colloids Surf., A, 2000, 173, 1-38.

20 M. J. Hampden-Smith, C. J. Brinker and W. G. Klemperer, Better ceramics through chemistry, Materials Research Society, 1992.

21 V. D. Land, T. M. Harris and D. C. Teeters, J. Non-Cryst. Solids, 2001, 283, 11-17.

22 F. Shi, L. Wang and J. Liu, Mater. Lett., 2006, 60, 3718-3722.

23 F. Schwertfeger, D. Frank and M. Schmidt, J. Non-Cryst. Solids, 1998, 225, 24-29.

24 A. P. Rao, A. V. Rao and G. Pajonk, Appl. Surf. Sci., 2007, 253, 6032-6040.

25 P. B. Sarawade, J.-K. Kim, A. Hilonga, D. V. Quang, S. J. Jeon and H. T. Kim, J. Non-Cryst. Solids, 2011, 357, 2156-2162.

26 Z. Li, X. Cheng, L. Shi, S. He, L. Gong, C. Li and H. Zhang, J. Hazard. Mater., 2016, 320, 350-358.

27 D. A. Loy and K. J. Shea, Chem. Rev., 1995, 95, 1431-1442.

28 A. V. Rao, M. M. Kulkarni, D. P. Amalnerkar and T. Seth, Appl. Surf. Sci., 2003, 206, 262-270.

29 A. Venkateswara Rao, M. M. Kulkarni, D. P. Amalnerkar and T. Seth, J. Non-Cryst. Solids, 2003, 330, 187-195.

30 F. Schwertfeger, W. Glaubitt and U. Schubert, J. Non-Cryst. Solids, 1992, 145, 85-89.

31 F. Schwertfeger, N. Hüsing and U. Schubert, J. Sol-Gel Sci. Technol., 1994, 2, 103-108.

32 Z. Wang, Z. Dai, J. Wu, N. Zhao and J. Xu, Adv. Mater., 2013, 25, 4494-4497.

33 Y. Lu, H. Fan, N. Doke, D. A. Loy, R. A. Assink, D. A. LaVan and C. J. Brinker, J. Am. Chem. Soc., 2000, 122, 5258-5261.

34 Z. Wang, D. Wang, Z. Qian, J. Guo, H. Dong, N. Zhao and J. Xu, ACS Appl. Mater. Interfaces, 2015, 7, 2016-2024.

35 S. Yun, H. Luo and Y. Gao, J. Mater. Chem. A, 2015, 3, 33903398.

36 A. Borba, M. Almangano, A. A. Portugal, R. Patricio and P. N. Simoes, J. Phys. Chem. A, 2016, 120, 4079-4088. 
37 S. Yun, H. Luo and Y. Gao, RSC Adv., 2014, 4, 4535-4542.

38 X. Guo, W. Li, H. Yang, K. Kanamori, Y. Zhu and K. Nakanishi, J. Sol-Gel Sci. Technol., 2013, 67, 406-413.

39 X. Guo, H. Yu, H. Yang, K. Kanamori, Y. Zhu and K. Nakanishi, J. Porous Mater., 2013, 20, 1477-1483.

40 K. Kanamori, M. Aizawa, K. Nakanishi and T. Hanada, Adv. Mater., 2007, 19, 1589-1593.

41 K. Kanamori, M. Aizawa, K. Nakanishi and T. Hanada, J. SolGel Sci. Technol., 2008, 48, 172-181.

42 K. Kanamori, K. Nakanishi and T. Hanada, Mater. Res. Soc. Symp. Proc., 2009, 1134, 173-178.

43 T. Shimizu, K. Kanamori and K. Nakanishi, Chem.-Eur. J., 2017, 23, 5176-5187.

44 T. Shimizu, K. Kanamori, A. Maeno, H. Kaji, C. M. Doherty, P. Falcaro and K. Nakanishi, Chem. Mater., 2016, 28, 68606868.

45 J. Yang, S. Mei and J. M. Ferreira,J. Am. Ceram. Soc., 2001, 84, 1696-1702.

46 J. L. Gurav, A. V. Rao, A. P. Rao, D. Y. Nadargi and S. D. Bhagat, J. Alloys Compd., 2009, 476, 397-402.
47 G.-Y. Jung, Z. Li, W. Wu, Y. Chen, D. L. Olynick, S.-Y. Wang, W. M. Tong and R. S. Williams, Langmuir, 2005, 21, 11581161.

48 L.-R. Bao, X. Cheng, X. Huang, L. Guo, S. Pang and A. Yee, J. Vac. Sci. Technol., B: Microelectron. Nanometer Struct.Process., Meas., Phenom., 2002, 20, 2881-2886.

49 E. K. Yim, R. M. Reano, S. W. Pang, A. F. Yee, C. S. Chen and K. W. Leong, Biomaterials, 2005, 26, 5405-5413.

50 A. Ershad-Langroudi, C. Mai, G. Vigier and R. Vassoille, J. Appl. Polym. Sci., 1997, 65, 2387-2393.

51 J. Liu, Z. Janjua, M. Roe, F. Xu, B. Turnbull, K.-S. Choi and X. Hou, Nanomaterials, 2016, 6, 232.

52 I. Vilaro, J. L. Yague and S. Borros, ACS Appl. Mater. Interfaces, 2017, 9, 1057-1065.

53 H. Budunoglu, A. Yildirim, M. O. Guler and M. Bayindir, ACS Appl. Mater. Interfaces, 2011, 3, 539-545.

54 S. Zong, W. Wei, Z. Jiang, Z. Yan, J. Zhu and J. Xie, RSC Adv., 2015, 5, 55579-55587. 